ANNALES

POLONICI MATHEMATICI

$94.3(2008)$

\title{
Infinite systems of first order PFDEs with mixed conditions
}

\author{
by W. Czernous (Gdańsk)
}

\begin{abstract}
We consider mixed problems for infinite systems of first order partial functional differential equations. An infinite number of deviating functions is permitted, and the delay of an argument may also depend on the spatial variable. A theorem on the existence of a solution and its continuous dependence upon initial boundary data is proved. The method of successive approximations is used in the existence proof. Infinite differential systems with deviated arguments and differential integral systems can be derived from the general model by specializing the operators.
\end{abstract}

1. Introduction. For any normed linear spaces $X$ and $Y$ and for $M \subset X$, let $C(M, Y)$ denote the class of all continuous functions from $M$ into $Y$. Inequalities between vectors will be understood componentwise.

Let $a>0, h_{0} \in \mathbb{R}_{+}, \mathbb{R}_{+}=[0, \infty), h=\left(h_{1}, \ldots, h_{n}\right)$, and $b=\left(b_{1}, \ldots, b_{n}\right)$ $\in \mathbb{R}^{n}$ be given where $b_{j}>0$ for $1 \leq j \leq n$. We define

$$
E=[0, a] \times[-b, b], \quad D=\left[-h_{0}, 0\right] \times[-h, h] .
$$

Let $\bar{c}=\left(c_{1}, \ldots, c_{n}\right)=b+h$ and

$$
\begin{aligned}
E_{0} & =\left[-h_{0}, 0\right] \times[-\bar{c}, \bar{c}], & \partial_{0} E & =[0, a] \times([-\bar{c}, \bar{c}] \backslash(-b, b)), \\
E^{*} & =E_{0} \cup E \cup \partial_{0} E, & \Omega & =E \times C(D, X) \times \mathbb{R}^{n} .
\end{aligned}
$$

Moreover, for $\tau \in(0, a]$ and a set $U \subset \mathbb{R}^{1+n}$ we set

$$
U_{\tau}=U \cap\left((-\infty, \tau] \times \mathbb{R}^{n}\right) .
$$

Suppose that $z: E^{*} \rightarrow \mathbb{R}$ and $(t, x) \in E$ are fixed. We define the function $z_{(t, x)}: D \rightarrow \mathbb{R}$ by

$$
z_{(t, x)}(\zeta, \xi)=z(t+\zeta, x+\xi), \quad(\zeta, \xi) \in D .
$$

The function $z_{(t, x)}$ is the restriction of $z$ to $\left[t-h_{0}, t\right] \times[x-h, x+h]$ and this restriction is shifted to $D$.

2000 Mathematics Subject Classification: 35R10, 35L60.

Key words and phrases: infinite systems, bicharacteristics, classical solutions, Volterra type operators. 
Let $\mathcal{S}$ be an arbitrary set of indices and let

$$
X=\left\{p=\left\{p_{s}\right\}_{s \in \mathcal{S}}: p_{s} \in \mathbb{R} \text { for } s \in \mathcal{S} \text { and }\|p\|=\sup \left\{\left|p_{s}\right|: s \in \mathcal{S}\right\}<\infty\right\} .
$$

We will denote by $M_{m \times k}$ the class of all $m \times k$ real matrices. For $y=$ $\left(y_{1}, \ldots, y_{m}\right) \in \mathbb{R}^{m}$ and $A=\left[a_{i j}\right]_{i=1, \ldots, m, j=1, \ldots, k} \in M_{m \times k}$ we put

$$
\|y\|=\sum_{j=1}^{m}\left|y_{j}\right| \text { and }\|A\|=\max _{1 \leq j \leq k} \sum_{i=1}^{m}\left|a_{i j}\right| .
$$

The product of two matrices is denoted by "*", and the scalar product in $\mathbb{R}^{m}$ by "o". If $A \in M_{m \times k}$, then $A^{T}$ is the transpose matrix. We next define

$$
X^{m}=\left\{y=\left\{y_{s}\right\}_{s \in \mathcal{S}}: y_{s} \in \mathbb{R}^{m}, s \in \mathcal{S} ;\|y\|=\sup \left\{\left\|y_{s}\right\|: s \in \mathcal{S}\right\}<\infty\right\}
$$

and

$$
\begin{array}{r}
X^{m \times k}=\left\{A=\left\{A_{s}\right\}_{s \in \mathcal{S}}: A_{s} \in M_{m \times k}, s \in \mathcal{S} ;\right. \\
\left.\|A\|=\sup \left\{\left\|A_{s}\right\|: s \in \mathcal{S}\right\}<\infty\right\} .
\end{array}
$$

For $\eta \in X^{m}$ we will write $\left(\eta_{1, s}, \ldots, \eta_{m, s}\right)=\eta_{s}$. Let $\|\cdot\|_{\xi},\|\cdot\|_{(\xi)},\|\cdot\|_{D}$ denote the supremum norms in $C\left(E_{\xi}^{*}, X\right), C\left(E_{\xi}, X^{m}\right), C(D, X)$, respectively. For a domain $U \subset \mathbb{R}^{1+n}$ and for functions $z: U \rightarrow X, u: U \rightarrow \mathbb{R}^{n}$ of the variables $(t, x)$ we will write

$$
\begin{gathered}
\partial_{t} z=\left\{\partial_{t} z_{s}\right\}_{s \in \mathcal{S}}, \quad \partial_{x} z=\left\{\partial_{x} z_{s}\right\}_{s \in \mathcal{S}}=\left\{\left(\partial_{x_{1}} z_{s}, \ldots, \partial_{x_{n}} z_{s}\right)\right\}_{s \in \mathcal{S}}, \\
\partial z=\left(\partial_{t} z, \partial_{x} z\right), \quad \partial_{t} u=\left(\partial_{t} u_{1}, \ldots, \partial_{t} u_{n}\right)^{T}, \quad \partial_{x} u=\left[\partial_{x_{j}} u_{i}\right]_{i, j=1, \ldots, n},
\end{gathered}
$$

provided that the derivatives exist.

Let

$$
\begin{gathered}
f: E^{*} \times C(D, X) \times \mathbb{R}^{n} \rightarrow X, \quad \varphi: E_{0} \cup \partial_{0} E \rightarrow X, \\
\alpha_{0}=\left\{\alpha_{0, s}\right\}_{s \in \mathcal{S}}: E \rightarrow X, \quad \alpha^{\prime}: E \rightarrow X^{n}
\end{gathered}
$$

be given functions. We write $\alpha_{s}=\left(\alpha_{0, s}, \alpha_{s}^{\prime}\right)$. For a function $z: E^{*} \rightarrow X$ and a point $(t, x) \in E$, we write

$$
z_{\alpha(t, x)}=\left\{\left(z_{s}\right)_{\alpha_{s}(t, x)}\right\}_{s \in \mathcal{S}} .
$$

We consider the system of functional differential equations

$$
\partial_{t} z_{s}(t, x)=f_{s}\left(t, x, z_{\alpha(t, x)}, \partial_{x} z_{s}(t, x)\right), \quad s \in \mathcal{S},
$$

with the initial boundary condition

$$
z(t, x)=\varphi(t, x) \quad \text { on } E_{0} \cup \partial_{0} E .
$$

For $\xi \in \mathbb{R}, 0<\xi \leq a$, we define a classical solution $\widetilde{z}: E_{\xi}^{*} \rightarrow X$ of the system (1), (2) to be a continuous function satisfying the system (1) on $E$, the condition (2), and having the derivatives $\partial_{t} \widetilde{z}_{s}, \partial_{x} \widetilde{z}_{s}, s \in \mathcal{S}$, at every point of $E$. In fact, higher regularity of solutions is proved in this paper (see p. 213 for the definition of the relevant function space). 
Note that our hereditary setting contains well known delay structures as particular cases.

ExAmple 1.1. Suppose that $\tilde{f}: E \times X \times \mathbb{R}^{n} \rightarrow X$ is a given function. Set $f_{s}(t, x, w, q)=\widetilde{f}_{s}(t, x, w(0,0), q), s \in \mathcal{S}$, then

$$
f_{s}\left(t, x, z_{\alpha(t, x)}, \partial_{x} z_{s}(t, x)\right)=\widetilde{f}_{s}\left(t, x, z(\alpha(t, x)), \partial_{x} z_{s}(t, x)\right), \quad s \in \mathcal{S},
$$

and (1) becomes a system with deviated variables.

EXAmple 1.2. For a function $w \in C(D, X)$ and a measurable set $B \subset D$ write $\int_{B} w(\xi, y) d y d \xi=\left\{\int_{B} w_{s}(\xi, y) d y d \xi\right\}_{s \in \mathcal{S}}$. For the above $\widetilde{f}$ we put

$$
f_{s}(t, x, w, q)=\widetilde{f}_{s}\left(t, x, \int_{B} w(\xi, y) d y d \xi, q\right), \quad s \in \mathcal{S} .
$$

Then

$$
f_{s}\left(t, x, z_{\alpha(t, x)}, \partial_{x} z_{s}(t, x)\right)=\widetilde{f}_{s}\left(t, x, \int_{B} z_{\alpha(\xi, y)} d y d \xi, \partial_{x} z_{s}(t, x)\right), \quad s \in \mathcal{S}
$$

and (1) becomes a differential integral system.

We will discuss the existence of solutions of problem (1), (2).

In recent years, numerous papers have been published, concerning first order partial functional differential equations. The following subjects have been investigated: functional differential inequalities and their applications, uniqueness of solutions to initial or initial boundary value problems, existence of classical or generalized solutions, numerical methods for functional differential equations. It is not our aim to give a full review of papers concerning the above problems. We just mention some results on the existence of solutions and cite only a few items of the vast literature.

Nonlinear evolutionary equations with first order partial derivatives have the following property: any classical solutions to initial or initial boundary value problem exist locally with respect to the time variable. This leads in a natural way to weak or generalized solutions.

Continuous solutions satisfying integral systems obtained by integrating original equations along bicharacteristics are considered in [12]. The Schauder fixed point theorem is used to prove existence results. That paper initiated the theory of first order partial functional differential equations.

Existence results for initial value problems for equations with deviated variables can be found in [1]. The Baiada method is applied there and the unknown functions depend on two variables. The paper [6] brings an existence result for quasilinear differential integral equations. The proof is based on the method of bicharacteristics.

Carathéodory solutions of quasilinear differential functional systems with initial or initial boundary conditions are investigated in [5], [16]. The method of bicharacteristics and functional integral inequalities are used in existence 
proofs. Weak solutions in the Cinquini Cibrario sense are studied in [3], [11]. Existence results for nonlinear equations are obtained by using the method of quasilinearization. It consists in constructing a quasilinear system for the unknown function and for its spatial derivatives. This system is then transformed into a system of integral functional equations of Volterra type. Continuous solutions of this integral problem lead to weak solutions of the original differential problem. Existence of Carathéodory solutions for nonlinear equations is considered in [4]; the constructive proof is based on the finite difference method. Classical solutions of nonlinear functional differential problems are considered in [2] and [8] (Chapter 2). These studies involve the method of successive approximations, introduced in [17] for systems without functional dependence. On each step of this method, differential problems without functional dependence arise. The existence of a sequence of successive approximations follows from a classical theorem. Partial differential inequalities are used in the proof of convergence of the successive approximations.

The work [14] initiated the theory of infinite systems of first order partial differential functional equations. Sufficient conditions are given in [14] for the existence of classical solutions to a generalized Cauchy problem

$$
\left\{\begin{array}{c}
\partial_{t} z_{i}(t, x)=G_{i}\left(t, x, z, \partial_{x} z_{i}(t, x)\right), \quad i=0,1,2, \ldots \\
z_{i}\left(a_{i}, x\right)=\chi_{i}(x) \text { for } x \in \mathbb{R}^{n},
\end{array}\right.
$$

The variable $z$ represents the functional argument in the system. The proof of the existence of solution is based on the following idea. A set $X_{c}$ is constructed, which is a closed subset of the Banach space consisting of sequences $z=\left\{z_{i}\right\}_{i=0}^{\infty}$ of the bounded continuous functions $z_{i}:[-c, c] \times \mathbb{R}^{n} \rightarrow \mathbb{R}$. For $u \in X_{c}$, consider the classical Cauchy problems

$$
\partial_{t} z_{i}(t, x)=G_{i}\left(t, x, u, \partial_{x} z_{i}(t, x)\right), \quad z_{i}\left(a_{i}, x\right)=\chi_{i}(x), \quad x \in \mathbb{R}^{n},
$$

where $i=0,1, \ldots$ Let us denote by $T u=\left\{T_{i} u\right\}_{i=0}^{\infty}$ the solution of (5). The set $X_{c}$ has the following property: $T u \in X_{c}$ and $T$ has exactly one fixed point $\widetilde{u} \in X_{c}$. This $\widetilde{u}$ is a classical solution of (5).

It is clear that the result from [14] can be extended to initial boundary value problems. The above existence result can be characterized as follows: the theorem has simple assumptions and the proof is very natural. Unfortunately, only a small class of problems are covered by this theorem. It is not applicable to differential problems with deviated variables nor to differential integral systems with right-hand sides given by (3).

Note that we have different models of functional dependence in (1) and (3). There are existence results for nonlinear problems ([2], [13], [16]) where the right-hand sides of the equations are superpositions of functions defined on a finite-dimensional Euclidean space and operators of Volterra 
type. The main assumptions in the existence theorems concern the operators of Volterra type, and are inequalities for norms in function spaces. Comparisons between different models of functional dependence are presented in [7].

Functional differential inequalities generated by infinite systems are investigated in [9], [15]. Uniqueness of solutions and continuous dependence on given functions are consequences of comparison theorems.

For further bibliography on partial differential functional equations and applications, see the monographs [8], [18].

This paper continues the author's study [3] of hyperbolic differentialfunctional equations, and generalizes some results of [10]. We now consider a more general form of functional dependence. Our approach admits equations with deviated argument, where the delay depends on all variables, not only on $t$, as was the case in former studies.

The paper is organized as follows. The notion of bicharacteristics for problem (1), (2) and their properties are presented in Section 2. Then the initial boundary value problem is transformed into a system of integral functional equations. This system is solved in Section 3 by the method of successive approximations. The main existence result and continuous dependence of solutions on the initial boundary functions are proved in Section 4 .

2. Bicharacteristics. The following function spaces will be needed in our considerations.

Given $s=\left(s_{1}, s_{2}\right) \in \mathbb{R}_{+}^{2}$, we denote by $C^{1 . L}[s]$ the set of all functions $\varphi \in C\left(E_{0} \cup \partial_{0} E, X\right)$ such that

$$
\begin{aligned}
\left|\partial_{t} \varphi(t, x)\right|+\left\|\partial_{x} \varphi(t, x)\right\| & \leq s_{1} \\
\left|\partial_{t} \varphi(t, x)-\partial_{t} \varphi(\bar{t}, \bar{x})\right|+\left\|\partial_{x} \varphi(t, x)-\partial_{x} \varphi(\bar{t}, \bar{x})\right\| & \leq s_{2}[|t-\bar{t}|+\|x-\bar{x}\|]
\end{aligned}
$$

on $E_{c}^{*}$.

For fixed $\varphi \in C^{1 . L}[s], 0<c \leq a$, and $d \in \mathbb{R}_{+}$with $d \geq s_{1}$, we consider the space $C_{\varphi . c}^{L}[d]$ of all $z \in C\left(E_{c}^{*}, X\right)$ such that $z(t, x)=\varphi(t, x)$ on $E_{0} \cup \partial_{0} E$ and

$$
\|z(t, x)-z(\bar{t}, \bar{x})\| \leq d[|t-\bar{t}|+\|x-\bar{x}\|] \quad \text { on } E_{c}^{*} .
$$

Let $p=\left(p_{0}, p_{1}\right) \in \mathbb{R}_{+}^{2}$ with $p_{0} \geq s_{1}, p_{1} \geq s_{2}$. We denote by $C_{\varphi . c}^{L}[p ; 1]$ the class of all functions $u_{0}: E_{c}^{*} \rightarrow X$ such that $u_{0}=\partial_{t} \varphi$ on $E_{0} \cup \partial_{0} E$ and

$$
\left\|u_{0}(t, x)\right\| \leq p_{0} \quad \text { and } \quad\left\|u_{0}(t, x)-u_{0}(\bar{t}, \bar{x})\right\| \leq p_{1}[|t-\bar{t}|+\|x-\bar{x}\|]
$$

on $E_{c}$.

Similarly, for such $p$, we denote by $C_{\varphi . c}^{L}[p ; n]$ the class of all functions $u: E_{c}^{*} \rightarrow X^{n}$ such that $u=\partial_{x} \varphi$ on $E_{0} \cup \partial_{0} E$ and 
$\|u(t, x)\| \leq p_{0} \quad$ and $\quad\|u(t, x)-u(\bar{t}, \bar{x})\| \leq p_{1}[|t-\bar{t}|+\|x-\bar{x}\|] \quad$ on $E_{c}$.

Write $C_{\varphi . c}^{L}[p ; 1+n]=C_{\varphi . c}^{L}[p ; 1] \times C_{\varphi . c}^{L}[p ; n]$. We prove that, under suitable assumptions on $f, \alpha$ and $\varphi$ and for sufficiently small $c$, there exists a solution $\bar{z}$ of problem (1), (2) such that $\bar{z} \in C_{\varphi . c}^{L}[d]$ and $\partial \bar{z} \in C_{\varphi . c}^{L}[p ; 1+n]$.

We begin with assumptions on $f$. For $1 \leq j \leq n$, define

$$
\begin{aligned}
\Delta_{+}^{(j)} & =\left\{(t, x) \in E: x_{j}=b_{j}\right\}, \quad \Delta_{-}^{(j)}=\left\{(t, x) \in E: x_{j}=-b_{j}\right\}, \\
\Delta & =\bigcup_{j=1}^{n}\left(\Delta_{+}^{(j)} \cup \Delta_{-}^{(j)}\right) .
\end{aligned}
$$

Assumption $\mathrm{H}\left[\partial_{q} f\right]$. The functions $f_{s}: \Omega \rightarrow \mathbb{R}, s \in \mathcal{S}$, of variables $(t, x, w, q)$ are continuous and satisfy the following conditions:

1) the partial derivatives

$$
\left(\partial_{q_{1}} f_{s}(P), \ldots, \partial_{q_{n}} f_{s}(P)\right)=\partial_{q} f_{s}(P), \quad P=(t, x, w, q), \quad s \in \mathcal{S},
$$

exist for $(t, x, w, q) \in \Omega$;

2) the functions $\partial_{q} f_{s}: \Omega \rightarrow \mathbb{R}^{n}, s \in \mathcal{S}$, are continuous and there are $B$, $L \in \mathbb{R}_{+}$such that

$$
\left\|\partial_{q} f(t, x, w, q)\right\| \leq B \quad \text { on } \Omega
$$

and

$\left\|\partial_{q} f(t, x, w, q)-\partial_{q} f(t, \bar{x}, \bar{w}, \bar{q})\right\| \leq L\left[\|x-\bar{x}\|+\|w-\bar{w}\|_{D}+\|q-\bar{q}\|\right] ;$

3) there is $\kappa>0$ such that for $1 \leq j \leq n$ and $s \in \mathcal{S}$ we have

$$
\begin{array}{ll}
\partial_{q_{j}} f_{s}(t, x, w, q) \geq 2 \kappa & \text { on }[0, a] \times \Delta_{+}^{(j)} \times C(D, X) \times \mathbb{R}^{n}, \\
\partial_{q_{j}} f_{s}(t, x, w, q) \leq-2 \kappa & \text { on }[0, a] \times \Delta_{-}^{(j)} \times C(D, X) \times \mathbb{R}^{n} .
\end{array}
$$

Assumption $\mathrm{H}[\alpha]$. The functions $\alpha_{s}: E \rightarrow E, s \in \mathcal{S}$, satisfy the following conditions:

1) $0 \leq \alpha_{0, s}(t, x) \leq t$ for $s \in \mathcal{S}$ and there is $r_{0} \in \mathbb{R}_{+}$such that

$$
\|\alpha(t, x)-\alpha(\bar{t}, \bar{x})\| \leq r_{0}[|t-\bar{t}|+\|x-\bar{x}\|] ;
$$

2) the derivatives $\partial_{x} \alpha_{0, s}, \partial_{x} \alpha_{s}^{\prime}, s \in \mathcal{S}$, exist on $E$ and there is $r_{1} \in \mathbb{R}_{+}$ such that

$$
\left\|\partial_{x} \alpha(t, x)-\partial_{x} \alpha(t, \bar{x})\right\| \leq r_{1}\|x-\bar{x}\|,
$$

where $\partial_{x} \alpha=\left\{\partial_{x} \alpha_{s}\right\}_{s \in \mathcal{S}}$.

Suppose that $\varphi \in C^{1 . L}[s], z \in C_{\varphi . c}^{L}[d], u \in C_{\varphi . c}^{L}[p ; n]$. Fix $s \in \mathcal{S}$ and consider the Cauchy problem

$$
\eta^{\prime}(\tau)=-\partial_{q} f_{s}\left(\tau, \eta(\tau), z_{\alpha(\tau, \eta(\tau))}, u_{s}(\tau, \eta(\tau))\right), \quad \eta(t)=x,
$$

and denote by $g_{s}\left[z, u_{s}\right](\cdot, t, x)$ its solution in the classical sense. The function $g_{s}\left[z, u_{s}\right](\cdot, t, x)$ is the $s$ th bicharacteristic of system (1) corresponding to 
$\left(z, u_{s}\right)$. Let $I_{(t, x) ; s}$ be its domain, and let $\delta_{s}\left[z, u_{s}\right](t, x)$ denote the left end of the maximal interval on which the bicharacteristic is defined. The set of all bicharacteristics of (1) corresponding to $(z, u) \in C_{\varphi . c}^{L}[d] \times C_{\varphi . c}^{L}[p ; n]$ is denoted by $g[z, u]=\left\{g_{s}\left[z, u_{s}\right]\right\}_{s \in \mathcal{S}}$.

Let

$$
\begin{aligned}
P_{s}\left[z, u_{s}\right] & (\tau, t, x) \\
= & \left(\tau, g_{s}\left[z, u_{s}\right](\tau, t, x), z_{\alpha\left(\tau, g_{s}\left[z, u_{s}\right](\tau, t, x)\right)}, u_{s}\left(\tau, g_{s}\left[z, u_{s}\right](\tau, t, x)\right)\right) .
\end{aligned}
$$

We prove a lemma on bicharacteristics.

Lemma 2.1. Suppose that Assumptions $\left[\mathrm{H} \partial_{q} f\right]$ and $\mathrm{H}[\alpha]$ are satisfied and $\varphi, \bar{\varphi} \in C^{1 . L}[s], z \in C_{\varphi . c}^{L}[d], \bar{z} \in C_{\bar{\varphi} . c}^{L}[d], u \in C_{\varphi . c}^{L}[p ; n], \bar{u} \in C_{\bar{\varphi} . c}^{L}[p ; n]$ are given. Then for each $s \in \mathcal{S}$ the solutions $g_{s}\left[z, u_{s}\right](\cdot, t, x)$ and $g_{s}\left[\bar{z}, \bar{u}_{s}\right](\cdot, t, x)$ exist on the intervals $I_{(t, x) ; s}$ and $\bar{I}_{(t, x) ; s}$, respectively, such that, for $\xi=$ $\delta_{s}\left[z, u_{s}\right](t, x)$ and $\bar{\xi}=\delta_{s}\left[\bar{z}, \bar{u}_{s}\right](t, x)$, we have $g_{s}\left[z, u_{s}\right](\xi, t, x) \in \Delta$ and $g_{s}\left[\bar{z}, \bar{u}_{s}\right](\bar{\xi}, t, x) \in \Delta$. The bicharacteristics are unique on $I_{(t, x) ; s}$ and $\bar{I}_{(t, x) ; s}$. Moreover,

$$
\begin{gathered}
\left\|g_{s}\left[z, u_{s}\right](\tau, t, x)-g_{s}\left[z, u_{s}\right](\tau, \bar{t}, \bar{x})\right\| \leq \bar{C}[|t-\bar{t}|+\|x-\bar{x}\|] \\
\left\|g_{s}\left[z, u_{s}\right](\tau, t, x)-g_{s}\left[\bar{z}, \bar{u}_{s}\right](\tau, t, x)\right\| \leq \bar{C}\left|\int_{t}^{\tau}\left[\|z-\bar{z}\|_{\xi}+\|u-\bar{u}\|_{(\xi)}\right] d \xi\right|
\end{gathered}
$$

where $\bar{C}=\max \{1, B, L\} \exp (c L \bar{d})$ and $\bar{d}=1+r_{0} d+p_{1}$. Furthermore, the functions $\delta_{s}\left[z, u_{s}\right](t, x)$ and $\delta_{s}\left[\bar{z}, \bar{u}_{s}\right](t, x)$ are continuous on $E_{c}$ and

$$
\begin{aligned}
& \left|\delta_{s}\left[z, u_{s}\right](t, x)-\delta_{s}\left[z, u_{s}\right](\bar{t}, \bar{x})\right| \leq \widehat{C}[|t-\bar{t}|+\|x-\bar{x}\|], \\
& \left|\delta_{s}\left[z, u_{s}\right](t, x)-\delta_{s}\left[\bar{z}, \bar{u}_{s}\right](t, x)\right| \leq \widehat{C} \int_{0}^{t}\left[\|z-\bar{z}\|_{\xi}+\|u-\bar{u}\|_{(\xi)}\right] d \xi,
\end{aligned}
$$

where $\widehat{C}=\bar{C} \kappa^{-1}$.

Proof. The existence and uniqueness of solutions of (6) follow from the classical theorems on the solutions of Cauchy problems. The function $g_{s}\left[z, u_{s}\right](\cdot, t, x)$ satisfies the integral equation

$$
g_{s}\left[z, u_{s}\right](\tau, t, x)=x-\int_{t}^{\tau} \partial_{q} f_{s}\left(P_{s}\left[z, u_{s}\right](\xi, t, x)\right) d \xi .
$$

Since $z \in C_{\varphi . c}^{L}[d]$, condition 2) of Assumption $\mathrm{H}[\alpha]$ shows that

$$
\left\|z_{\alpha(\tau, y)}-z_{\alpha(\tau, \bar{y})}\right\|_{D} \leq r_{0} d\|y-\bar{y}\|
$$


for all $(\tau, y),(\tau, \bar{y}) \in E_{c}$. It follows from Assumption $\mathrm{H}\left[\partial_{q} f\right]$ that

$$
\begin{aligned}
\left\|g_{s}\left[z, u_{s}\right](\tau, t, x)-g_{s}\left[z, u_{s}\right](\tau, \bar{t}, \bar{x})\right\| & \leq \max \{1, B\}[|t-\bar{t}|+\|x-\bar{x}\|] \\
& +L \bar{d}\left|\int_{t}^{\tau}\left\|g_{s}\left[z, u_{s}\right](\xi, t, x)-g_{s}\left[z, u_{s}\right](\xi, \bar{t}, \bar{x})\right\| d \xi\right|
\end{aligned}
$$

for $\tau \in I_{(t, x) ; s} \cap I_{(\bar{t}, \bar{x}) ; s}$. We now obtain (7) by the Gronwall inequality. For $(\tau, y),(\tau, \bar{y}) \in E_{c}$, we have

$$
\left\|z_{\alpha(\tau, y)}-\bar{z}_{\alpha(\tau, \bar{y})}\right\|_{D} \leq\|z-\bar{z}\|_{\tau}+r_{0} d\|y-\bar{y}\| .
$$

It follows that

$$
\begin{aligned}
&\left\|g_{s}\left[z, u_{s}\right](\tau, t, x)-g_{s}\left[\bar{z}, \bar{u}_{s}\right](\tau, t, x)\right\| \leq L\left|\int_{t}^{\tau}\left(\|z-\bar{z}\|_{\xi}+\|u-\bar{u}\|_{(\xi)}\right) d \xi\right| \\
&+L \bar{d}\left|\int_{t}^{\tau}\left\|g_{s}\left[z, u_{s}\right](\xi, t, x)-g_{s}\left[\bar{z}, \bar{u}_{s}\right](\xi, t, x)\right\| d \xi\right|
\end{aligned}
$$

for $\tau \in I_{(t, x) ; s} \cap \bar{I}_{(t, x) ; s}$, and we get (8) from the Gronwall inequality.

The continuity of $\delta_{s}\left[z, u_{s}\right]$ and $\delta_{s}\left[\bar{z}, \bar{u}_{s}\right]$ on $E_{c}$ follows from the classical theorems on continuous dependence of Cauchy problems' solutions on initial conditions. We now prove (9). This estimate is obvious if $\delta_{s}\left[z, u_{s}\right](t, x)=$ $\delta_{s}\left[z, u_{s}\right](\bar{t}, \bar{x})=0$ (i.e. the solutions of problem (6) are defined on $[0, t]$ and $[0, \bar{t}])$. Now assume that $0 \leq \delta_{s}\left[z, u_{s}\right](t, x)<\delta_{s}\left[z, u_{s}\right](\bar{t}, \bar{x})$. Then, for $\bar{\zeta}=$ $\delta_{s}\left[z, u_{s}\right](\bar{t}, \bar{x})$, we have $g_{s}\left[z, u_{s}\right](\bar{\zeta}, \bar{t}, \bar{x}) \in \Delta$ and there exists $i, 1 \leq i \leq n$, such that $\left|g_{i, s}(\bar{\zeta}, \bar{t}, \bar{x})\right|=b_{i}$. The following two cases are possible:

(i) $g_{i, s}(\bar{\zeta}, \bar{t}, \bar{x})=b_{i}$;

(ii) $g_{i, s}(\bar{\zeta}, \bar{t}, \bar{x})=-b_{i}$.

Consider case (i). Let $x=\left(x_{1}, \ldots, x_{n}\right), \widetilde{x}=\left(x_{1}, \ldots, x_{i-1}, b_{i}, x_{i+1}, \ldots, x_{n}\right)$. Then for $(t, x) \in E_{c}$,

$$
\left|\partial_{q_{i}} f_{s}\left(t, x, z_{\alpha(t, x)}, u(t, x)\right)-\partial_{q_{i}} f_{s}\left(t, \widetilde{x}, z_{\alpha(t, \widetilde{x})}, u_{s}(t, \widetilde{x})\right)\right| \leq \widetilde{c}\left(b_{i}-x_{i}\right),
$$

where $\widetilde{c}=L \bar{d}$. Thus

$$
\partial_{q_{i}} f_{s}\left(t, x, z_{\alpha(t, x)}, u_{s}(t, x)\right) \geq \kappa
$$

for $(t, x) \in E_{c}$ such that $b_{i}-x_{i} \leq \kappa \widetilde{c}^{-1}$. It follows from (7) that

$$
b_{i}-g_{i, s}\left[z, u_{s}\right](\bar{\zeta}, t, x)=g_{i, s}\left[z, u_{s}\right](\bar{\zeta}, \bar{t}, \bar{x})-g_{i, s}\left[z, u_{s}\right](\bar{\zeta}, t, x) \leq \kappa / \widetilde{c}
$$

for $(t, x),(\bar{t}, \bar{x}) \in E_{c}$ such that

$$
|t-\bar{t}|+\|x-\bar{x}\| \leq \kappa / \widetilde{c} \bar{C} .
$$

Hence

$$
\partial_{q_{i}} f_{s}\left(\bar{\zeta}, g_{s}\left[z, u_{s}\right](\bar{\zeta}, t, x), z_{\alpha\left(\bar{\zeta}, g_{s}\left[z, u_{s}\right](\bar{\zeta}, t, x)\right)}, u_{s}\left(\bar{\zeta}, g_{s}\left[z, u_{s}\right](\bar{\zeta}, t, x)\right)\right) \geq \kappa>0,
$$


and consequently

$$
\partial_{t} g_{i, s}\left[z, u_{s}\right]\left(\delta_{s}\left[z, u_{s}\right](\bar{t}, \bar{x}), t, x\right)<0
$$

for $(t, x),(\bar{t}, \bar{x}) \in E_{c}$ satisfying (13). It is easy to see that $g_{i, s}(\cdot, t, x)$ is decreasing on the interval $\left(\delta_{s}\left[z, u_{s}\right](t, x), \delta_{s}\left[z, u_{s}\right](\bar{t}, \bar{x})\right)$. Therefore

$$
b_{i}-g_{i, s}\left[z, u_{s}\right](\tau, t, x) \leq \kappa / \widetilde{c},
$$

and

$$
\begin{aligned}
& \partial_{q_{i}} f_{s}\left(P_{s}\left[z, u_{s}\right](\tau, t, x)\right) \\
& \quad=\partial_{q_{i}} f_{s}\left(\tau, g_{s}\left[z, u_{s}\right](\tau, t, x), z_{\alpha\left(\tau, g_{s}\left[z, u_{s}\right](\tau, t, x)\right)}, u_{s}\left(\tau, g_{s}\left[z, u_{s}\right](\tau, t, x)\right)\right) \geq \kappa
\end{aligned}
$$

for $\tau \in\left(\delta_{s}\left[z, u_{s}\right](t, x), \delta_{s}\left[z, u_{s}\right](\bar{t}, \bar{x})\right)$ and $(t, x),(\bar{t}, \bar{x}) \in E_{c}$ such that (13) is satisfied. Hence

$$
\begin{aligned}
-\kappa\left[\delta_{s}[z\right. & \left.\left., u_{s}\right](\bar{t}, \bar{x})-\delta_{s}\left[z, u_{s}\right](t, x)\right] \\
& \geq-\int_{\delta_{s}\left[z, u_{s}\right](t, x)}^{\delta_{s}\left[z, u_{s}\right](\bar{t}, \bar{x})} \partial_{q_{i}} f_{s}\left(P_{s}\left[z, u_{s}\right](\tau, t, x)\right) d \tau \\
& =g_{i, s}\left[z, u_{s}\right]\left(\delta_{s}\left[z, u_{s}\right](\bar{t}, \bar{x}), t, x\right)-g_{i, s}\left[z, u_{s}\right]\left(\delta_{s}\left[z, u_{s}\right](t, x), t, x\right) \\
& \geq g_{i, s}\left[z, u_{s}\right]\left(\delta_{s}\left[z, u_{s}\right](\bar{t}, \bar{x}), t, x\right)-g_{i, s}\left[z, u_{s}\right]\left(\delta_{s}\left[z, u_{s}\right](\bar{t}, \bar{x}), \bar{t}, \bar{x}\right) \\
& \geq-\bar{C}[|t-\bar{t}|+\|x-\bar{x}\|] .
\end{aligned}
$$

Thus, the proof of (9) for $(t, x),(\bar{t}, \bar{x}) \in E_{c}$ such that (13) holds is complete in case (i). In case (ii) we proceed in a similar way. Note that we have proved a local Lipschitz property of $\delta_{s}\left[z, u_{s}\right]$ with respect to $(t, x)$ with a Lipschitz constant independent of $(t, x)$. Hence $\delta_{s}\left[z, u_{s}\right]$ satisfies a global Lipschitz condition with respect to $(t, x)$ with the same constant. That proves $(9)$.

The proof of (10) is similar.

3. Integral functional equations. We denote by $C L(D, X)$ the set of all linear and continuous real functions defined on $C(D, X)$ and we denote by $\|\cdot\|$ the norm in $C L(D, X)$ generated by the supremum norm $\|\cdot\|_{D}$ in the space $C(D, X)$.

Assumption $\mathrm{H}[f]$. The Assumption $\mathrm{H}\left[\partial_{q} f\right]$ is satisfied and:

1) there is $B_{0} \in \mathbb{R}_{+}$such that $\|f(t, x, w, q)\| \leq B_{0}$ on $\Omega$,

2) for each $s \in \mathcal{S}$ the partial derivatives $\left(\partial_{x_{1}} f_{s}(P), \ldots, \partial_{x_{n}} f_{s}(P)\right)=$ $\partial_{x} f_{s}(P), P=(t, x, w, q)$, and the Fréchet derivative $\partial_{w} f_{s}(P) \in$ $C L(D, X)$ exist on $\Omega$,

3) the estimates $\left\|\partial_{x} f(t, x, w, q)\right\| \leq B$ and

$$
\left\|\partial_{w} f(t, x, w, q)\right\|=\sup \left\{\left\|\partial_{w} f_{s}(t, x, w, q)\right\|: s \in \mathcal{S}\right\} \leq B
$$

are satisfied on $\Omega$, where $\partial_{x} f=\left\{\partial_{x} f_{s}\right\}_{s \in \mathcal{S}}, \partial_{w} f=\left\{\partial_{w} f_{s}\right\}_{s \in \mathcal{S}}$, 
4) there is $B_{1} \in \mathbb{R}_{+}$such that for $t, \bar{t} \in[0, a]$ and $(x, w, q) \in \mathbb{R}^{n} \times$ $C(D, X) \times \mathbb{R}^{n}$ we have

$$
\|f(t, x, w, q)-f(\bar{t}, x, w, q)\| \leq B_{1}|t-\bar{t}|,
$$

5) for $(t, x, w, q) \in \Omega$ and $\bar{w} \in C(D, X)$ we have

$$
\begin{aligned}
\left\|\partial_{x} f(t, x, w, q)-\partial_{x} f(t, \bar{x}, \bar{w}, \bar{q})\right\| & \leq L\left[\|x-\bar{x}\|+\|w-\bar{w}\|_{D}+\|q-\bar{q}\|\right], \\
\left\|\partial_{w} f(t, x, w, q)-\partial_{w} f(t, \bar{x}, \bar{w}, \bar{q})\right\| & \leq L\left[\|x-\bar{x}\|+\|w-\bar{w}\|_{D}+\|q-\bar{q}\|\right] .
\end{aligned}
$$

REMARK 3.1. We will give a theorem on the existence of solutions of problem (1), (2). For simplicity of formulation, we have assumed the same estimates for the derivatives $\partial_{x} f, \partial_{w} f$ and $\partial_{q} f$. We have also assumed the Lipschitz condition for these derivatives with the same coefficient.

We now exhibit a system of integral equations generated by (1), (2). We write

$$
\begin{aligned}
Q_{s}\left[z, u_{s}\right](t, x) & =\left(\delta_{s}\left[z, u_{s}\right](t, x), g_{s}\left[z, u_{s}\right]\left(\delta_{s}\left[z, u_{s}\right](t, x), t, x\right)\right), \\
\Phi_{s}\left[z, u_{s}\right](t, x) & =\varphi_{s}\left(Q\left[z, u_{s}\right](t, x)\right), \\
\Psi_{s}\left[z, u_{s}\right](t, x) & =\partial_{x} \varphi_{s}\left(Q\left[z, u_{s}\right](t, x)\right), \\
\bar{W}_{s}\left[u_{0, s}, u_{s}\right](t, x) & =\left(u_{0, s}, u_{s}\right)_{\alpha_{s}(t, x)} * \partial_{x} \alpha_{s}(t, x), \\
\bar{W}\left[u_{0}, u\right](t, x) & =\left\{\bar{W}_{s}\left[u_{0, s}, u_{s}\right](t, x)\right\}_{s \in \mathcal{S}}, \\
W_{\{s\}}\left[z, u_{0}, u\right](\tau, t, x) & =\bar{W}\left[u_{0}, u\right]\left(\tau, g_{s}\left[z, u_{s}\right](\tau, t, x)\right) \in C(D, X) .
\end{aligned}
$$

For $u \in C\left(D, X^{n}\right), P \in \Omega$ and $s \in \mathcal{S}$, we set

$$
\partial_{w} f_{s}(P) u=\left(\partial_{w} f_{s}(P) u_{1}, \ldots, \partial_{w} f_{s}(P) u_{n}\right) .
$$

Given $\varphi \in C^{1 . L}[s], z \in C_{\varphi . c}^{L}[d]$ and $u_{0} \in C_{\varphi . c}^{L}[p ; 1], u \in C_{\varphi . c}^{L}[p ; n]$, where $0<c \leq a$, we define

$$
\begin{aligned}
F_{s}\left[z, u_{s}\right](t, x)= & \Phi_{s}\left[z, u_{s}\right](t, x) \\
& +\int_{\delta_{s}\left[z, u_{s}\right](t, x)}^{t}\left[f_{s}\left(P_{s}\left[z, u_{s}\right](\tau, t, x)\right)\right. \\
& \left.-u\left(\tau, g_{s}\left[z, u_{s}\right](\tau, t, x)\right) \circ \partial_{q} f_{s}\left(P_{s}\left[z, u_{s}\right](\tau, t, x)\right)\right] d \tau \\
G_{s}\left[z, u_{0}, u\right](t, x)= & \Psi_{s}\left[z, u_{s}\right](t, x) \\
& +\int_{\delta_{s}\left[z, u_{s}\right](t, x)}^{t}\left[\partial_{x} f_{s}\left(P_{s}\left[z, u_{s}\right](\tau, t, x)\right)\right. \\
& \left.+\partial_{w} f_{s}\left(P_{s}\left[z, u_{s}\right](\tau, t, x)\right) W_{\{s\}}\left[z, u_{0}, u\right](\tau, t, x)\right] d \tau
\end{aligned}
$$

for $s \in \mathcal{S}$. Write

$$
F[z, u]=\left\{F_{s}\left[z, u_{s}\right]\right\}_{s \in \mathcal{S}} \quad \text { and } \quad G\left[z, u_{0}, u\right]=\left\{G_{s}\left[z, u_{0}, u\right]\right\}_{s \in \mathcal{S}} .
$$


Consider the system of integral functional equations

$$
z(t, x)=F[z, u](t, x), \quad u(t, x)=G\left[z, u_{0}, u\right](t, x),
$$

where

$$
u_{0, s}(t, x)=f_{s}\left(t, x, z_{\alpha(t, x)}, u_{s}(t, x)\right), \quad s \in \mathcal{S},
$$

with the initial boundary condition

$$
z=\varphi, \quad\left(u_{0}, u\right)=\partial \varphi \quad \text { on } E_{0} \cup \partial_{0} E .
$$

REMARK 3.2. The integral functional system (16)-(18) is obtained in the following way. We first introduce an additional unknown function $u$, where $u=\partial_{x} z$. Then we consider the linearization of (1) with respect to $u$, i.e.

$$
\partial_{t} z_{s}(t, x)=f_{s}\left(U_{s}\right)+\partial_{q} f_{s}\left(U_{s}\right) \circ\left(\partial_{x} z_{s}(t, x)-u_{s}(t, x)\right), \quad s \in \mathcal{S},
$$

where $U_{s}=\left(t, x, z_{\alpha(t, x)}, u_{s}(t, x)\right)$. By virtue of equation (1) we get a differential system for the unknown function $u$ :

$$
\begin{aligned}
{\left[\partial_{t} u_{s}(t, x)\right]^{T}=} & \partial_{x} f_{s}\left(U_{s}\right)+\partial_{q} f_{s}\left(U_{s}\right) *\left[\partial_{x} u_{s}(t, x)\right]^{T} \\
& +\partial_{w} f\left(U_{s}\right) \bar{W}\left[\partial_{t} z, \partial_{x} z\right](t, x), \quad s \in \mathcal{S},
\end{aligned}
$$

where $\partial_{t} z=\left\{\partial_{t} z_{s}\right\}_{s \in \mathcal{S}}, \partial_{x} z=\left\{\partial_{x} z_{s}\right\}_{s \in \mathcal{S}}$. Finally, we put $\partial_{t} z=u_{0}$ and $\partial_{x} z=u$ in (20). System (19), (20) has the following property: the differential equations of bicharacteristics for (19) and for (20) are the same and they have the form (6).

If we consider (19), (20) along the bicharacteristics $g_{s}\left[z, u_{s}\right](\cdot, t, x)$, we obtain, for $s \in \mathcal{S}$,

$$
\begin{aligned}
& \frac{d}{d \tau} z_{s}\left(\tau, g_{s}\left[z, u_{s}\right](\tau, t, x)\right) \\
& \quad=f_{s}\left(P_{s}\left[z, u_{s}\right](\tau, t, x)\right)-\partial_{q} f_{s}\left(P_{s}\left[z, u_{s}\right](\tau, t, x)\right) \circ u_{s}\left(\tau, g_{s}\left[z, u_{s}\right](\tau, t, x)\right)
\end{aligned}
$$

and

$$
\begin{aligned}
\frac{d}{d \tau} u_{s} & \left(\tau, g_{s}\left[z, u_{s}\right](\tau, t, x)\right) \\
& =\partial_{x} f_{s}\left(P_{s}\left[z, u_{s}\right](\tau, t, x)\right)+\partial_{w} f_{s}\left(P_{s}\left[z, u_{s}\right](\tau, t, x)\right) W_{\{s\}}\left[z, u_{0}, u\right](\tau, t, x) .
\end{aligned}
$$

By integrating the above equations on $\left[\delta_{s}\left[z, u_{s}\right](t, x), t\right]$ with respect to $\tau$, we get (16). Again by virtue of equation (1), we get the formula (17) for $u_{0}$.

The existence results for (16)-(18) are based on the following method of successive approximations. Assume that $\varphi \in C^{1 . L}[s]$ and Assumption $\mathrm{H}[f]$ is satisfied. We define a sequence $\left\{z^{(m)}, u_{0}^{(m)}, u^{(m)}\right\}$ in the following way: Write $\left(z, u_{0}, u\right)^{(m)}=\left(z^{(m)}, u_{0}^{(m)}, u^{(m)}\right)$ and $\mathcal{C}=C_{\varphi . c}^{L}[d] \times C_{\varphi . c}^{L}[p ; 1] \times C_{\varphi . c}^{L}[p ; n]$. Let $\left(z, u_{0}, u\right)^{(0)} \in \mathcal{C}$ be arbitrary differentiable functions such that $\partial z^{(0)}=$ $\left(u_{0}^{(0)}, u^{(0)}\right)$ on $E_{c}$. Next, if $\left(z, u_{0}, u\right)^{(m)} \in \mathcal{C}$ are known functions, then $u^{(m+1)}$ 
is a solution of the equation

$$
u=G^{(m)}[u]
$$

and

$$
\left.\begin{array}{l}
z^{(m+1)}(t, x)=F\left[z^{(m)}, u^{(m+1)}\right](t, x) \\
u_{0, s}^{(m+1)}(t, x)=f_{s}\left(t, x, z_{\alpha(t, x)}^{(m)}, u_{s}^{(m+1)}(t, x)\right), \quad s \in \mathcal{S}
\end{array}\right\} \quad \text { on } E_{c}
$$

where $G^{(m)}=\left\{G_{s}^{(m)}\right\}_{s \in \mathcal{S}}$ is defined by

$$
\begin{aligned}
G_{s}^{(m)}[u](t, x)= & \Psi_{s}\left[z^{(m)}, u_{s}\right](t, x) \\
& +\int_{\delta_{s}\left[z^{(m)}, u_{s}\right](t, x)}^{t}\left[\partial_{x} f_{s}\left(P_{s}\left[z^{(m)}, u_{s}\right](\tau, t, x)\right)\right. \\
& \left.+\partial_{w} f_{s}\left(P_{s}\left[z^{(m)}, u_{s}\right](\tau, t, x)\right) W_{\{s\}}^{(m)}\left[u_{s}\right](\tau, t, x)\right] d \tau
\end{aligned}
$$

for $(t, x) \in E_{c}, s \in \mathcal{S}$, and by

$$
G^{(m)}[u](t, x)=\partial_{x} \varphi(t, x)
$$

for $(t, x) \in E_{0} \cup \partial_{0} E$, and where

$$
W_{\{s\}}^{(m)}\left[u_{s}\right](\tau, t, x)=\bar{W}\left[u_{0}^{(m)}, u^{(m)}\right]\left(\tau, g_{s}\left[z^{(m)}, u_{s}\right](\tau, t, x)\right) \in C(D, X) .
$$

We wish to emphasize that the main difficulty in carrying out this construction is to prove the existence of the sequence $\left\{z^{(m)}, u_{0}^{(m)}, u^{(m)}\right\}$.

We set $\bar{p}=1+n p_{0} r_{0}, \bar{\omega}=p_{1} r_{0}^{2}+p_{0} r_{1}, \bar{Q}=\bar{C}+\widehat{C}(1+B)$ and $A_{0}=$ $B \bar{p} \widehat{C}+c \bar{C}[L \bar{d} \bar{p}+n \bar{\omega} B]$.

Assumption $\mathrm{H}[c, d, p]$. The constants $c, d$ and $p=\left(p_{0}, p_{1}\right)$ satisfy the conditions

$$
\begin{aligned}
d= & p_{0} \geq \max \left\{s_{1}+c B \bar{p}, B_{0}\right\}, \\
& p_{1} \geq \max \left\{B \bar{p}+A_{0}+s_{2} \bar{Q}, B_{1}+B \bar{d}\right\} .
\end{aligned}
$$

It is easy to see that there are $d, p$, and $c \in(0, a]$ sufficiently small that satisfy Assumption $\mathrm{H}[c, d, p]$.

Lemma 3.3. If $\varphi \in C^{1 . L}[s]$ and Assumptions $\mathrm{H}[\alpha], \mathrm{H}[f]$ and $\mathrm{H}[c, d, p]$ are satisfied, then

$$
G^{(m)}: C_{\varphi . c}^{L}[p ; n] \rightarrow C_{\varphi . c}^{L}[p ; n] .
$$
Then

Proof. Suppose that $\varphi \in C^{1 . L}[s],\left(z, u_{0}, u\right)^{(m)} \in \mathcal{C}$ and $u \in C_{\varphi . c}^{L}[p ; n]$.

$$
\left\|G^{(m)}[u](t, x)\right\| \leq s_{1}+c B \bar{p} \quad \text { on } E_{c} .
$$


We now prove that the function $G^{(m)}[u]$ satisfies the Lipschitz condition with constant $p_{1}$. If $(t, x),(\bar{t}, \bar{x}) \in E_{c}$, then for each $s \in \mathcal{S}$,

$$
\left\|G_{s}^{(m)}[u](t, x)-G_{s}^{(m)}[u](\bar{t}, \bar{x})\right\| \leq A_{\varphi}+A_{f},
$$

where

$$
\begin{gathered}
A_{\varphi}=\left\|\partial_{x} \varphi_{s}\left(Q_{s}\left[z^{(m)}, u_{s}\right](t, x)\right)-\partial_{x} \varphi_{s}\left(Q_{s}\left[z^{(m)}, u_{s}\right](\bar{t}, \bar{x})\right)\right\|, \\
A_{f}=\| \int_{\delta_{s}\left[z^{(m)}, u_{s}\right](t, x)}^{t}\left[\partial_{x} f_{s}\left(P_{s}\left[z^{(m)}, u_{s}\right](\tau, t, x)\right)\right. \\
\left.\quad+\partial_{w} f_{s}\left(P_{s}\left[z^{(m)}, u_{s}\right](\tau, t, x)\right) W_{s}^{(m)}\left[u_{s}\right](\tau, t, x)\right] d \tau \\
-\int_{\delta_{s}\left[z^{(m)}, u_{s}\right](\bar{t}, \bar{x})}\left[\partial_{x} f_{s}\left(P_{s}\left[z^{(m)}, u_{s}\right](\tau, \bar{t}, \bar{x})\right)\right. \\
\left.\quad+\partial_{w} f_{s}\left(P_{s}\left[z^{(m)}, u_{s}\right](\tau, \bar{t}, \bar{x})\right) W_{s}^{(m)}\left[u_{s}\right](\tau, \bar{t}, \bar{x})\right] d \tau \| .
\end{gathered}
$$

It follows from Lemma 2.1 that

$$
A_{\varphi} \leq s_{2} \bar{Q}[|t-\bar{t}|+\|x-\bar{x}\|], \quad A_{f} \leq B \bar{p}|t-\bar{t}|+A_{0}[|t-\bar{t}|+\|x-\bar{x}\|] .
$$

Hence, using Assumption $\mathrm{H}[c, d, p]$, we get

$$
\left\|G^{(m)}[u](t, x)-G^{(m)}[u](\bar{t}, \bar{x})\right\| \leq p_{1}[|t-\bar{t}|+\|x-\bar{x}\|]
$$

on $E_{c}$. This inequality, together with (26) and (25), yields Lemma 3.3.

Lemma 3.4. Suppose that $\varphi \in C^{1 . L}[s]$ and Assumptions $\mathrm{H}[\alpha], \mathrm{H}[f]$, $\mathrm{H}[c, d, p]$ are satisfied. Then there exists exactly one function $\bar{u} \in C_{\varphi . c}^{L}[p ; n]$ satisfying the equation $u=G^{(m)}[u]$.

Proof. Lemma 3.3 shows that $G^{(m)}: C_{\varphi . c}^{L}[p ; n] \rightarrow C_{\varphi . c}^{L}[p ; n]$. It follows that there is $A>0$ such that, for $u, \bar{u} \in C_{\varphi . c}^{L}[p ; n]$, we have

$$
\left\|G^{(m)}[u](t, x)-G^{(m)}[\bar{u}](t, x)\right\| \leq A \int_{0}^{t}\|u-\bar{u}\|_{(\tau)} d \tau
$$

on $E_{c}$. We now define the norm in the space $C\left(E_{c}^{*}, X^{n}\right)$ as follows:

$$
\|u\|_{\lambda}=\max \left\{\|u(t, x)\| e^{-\lambda t}:(t, x) \in E_{c}^{*}\right\},
$$

where $\lambda>A$. It is easy to see that $\left(C\left(E_{c}^{*}, X^{n}\right),\|\cdot\|_{\lambda}\right)$ is a Banach space and $C_{\varphi . c}^{L}[p ; n]$ is its closed subset. Let us prove that there exists $q \in(0,1)$ such that

$$
\left\|G^{(m)}[u]-G^{(m)}[\bar{u}]\right\|_{\lambda} \leq q\|u-\bar{u}\|_{\lambda} \quad \text { for } u, \bar{u} \in C_{\varphi . c}^{L}[p ; n] .
$$


Since, from (25), $\left\|G^{(m)}[u](t, x)-G^{(m)}[\bar{u}](t, x)\right\| e^{-\lambda t}$ vanishes on $E_{0} \cup \partial_{0} E_{c}$, it is sufficient to estimate it on $E_{c}$. According to (27), we have

$$
\begin{aligned}
\| G^{(m)}[u](t, x)-G^{(m)}[\bar{u}](t, x) & \leq A \int_{0}^{t}\|u-\bar{u}\|_{(\xi)} d \xi \\
& =A \int_{0}^{t}\|u-\bar{u}\|_{(\xi)} e^{-\lambda \xi} e^{\lambda \xi} d \xi \leq A\|u-\bar{u}\|_{\lambda} \int_{0}^{t} e^{\lambda \xi} d \xi \\
& =\frac{A}{\lambda}\|u-\bar{u}\|_{\lambda}\left(e^{\lambda t}-1\right) \leq \frac{A}{\lambda}\|u-\bar{u}\|_{\lambda} e^{\lambda t}
\end{aligned}
$$

for $(t, x) \in E_{c}$. Hence

$$
\left\|G^{(m)}[u](t, x)-G^{(m)}[\bar{u}](t, x)\right\| e^{-\lambda t} \leq \frac{A}{\lambda}\|u-\bar{u}\|_{\lambda}, \quad(t, x) \in E_{c} .
$$

It follows that estimate (28) holds with $q=A \lambda^{-1}$. By the Banach fixedpoint theorem, there exists exactly one $\bar{u} \in C_{\varphi . c}^{L}[p ; n]$ satisfying the equation $u=G^{(m)}[u]$.

We now prove the main lemma in this section.

Lemma 3.5. If $\varphi \in C^{1 . L}[s]$ and Assumptions $\mathrm{H}[\alpha], \mathrm{H}[f]$ and $\mathrm{H}[c, d, p]$ are satisfied, then, for any $m \geq 0$, we have

$$
\partial z^{(m)}(t, x)=\left(u_{0}^{(m)}, u^{(m)}\right)(t, x) \quad \text { on } E_{c}
$$

and $z^{(m)} \in C_{\varphi . c}^{L}[d], u_{0}^{(m)} \in C_{\varphi . c}^{L}[p ; 1]$.

Proof. We prove (29) by induction. It follows from the definition of the sequence $\left\{\left(z, u_{0}, u\right)^{(m)}\right\}$ that $(29)$ is satisfied for $m=0$. Assuming that it holds for a given $m \geq 0$, we prove that $z^{(m+1)}, u_{0}^{(m)}$ given by (22) satisfy (29). We fix $s \in \mathcal{S}$ and set

$$
\begin{aligned}
\Delta^{(m)}(t, \bar{t}, x, \bar{x})= & z_{s}^{(m+1)}(\bar{t}, \bar{x})-z_{s}^{(m+1)}(t, x) \\
& -u_{0, s}^{(m+1)}(t, x)(\bar{t}-t)-u_{s}^{(m+1)}(t, x) \circ(\bar{x}-x),
\end{aligned}
$$

where $(t, x),(\bar{t}, \bar{x}) \in E_{c}$. We prove that there exists $\widetilde{C} \in \mathbb{R}_{+}$such that

$$
\left|\Delta^{(m)}(t, \bar{t}, x, \bar{x})\right| \leq \widetilde{C}\|\bar{x}-x\|^{2} \quad \text { on } E_{c} .
$$

Fix $(t, x),(\bar{t}, \bar{x}) \in E_{c}$ and set, for brevity,

$$
\begin{aligned}
g(\tau) & =g_{s}\left[z^{(m)}, u_{s}^{(m+1)}\right](\tau, t, x), & \bar{g}(\tau) & =g_{s}\left[z^{(m)}, u_{s}^{(m+1)}\right](\tau, \bar{t}, \bar{x}), \\
P(\tau) & =P_{s}\left[z^{(m)}, u_{s}^{(m+1)}\right](\tau, t, x), & \bar{P}(\tau) & =P_{s}\left[z^{(m)}, u_{s}^{(m+1)}\right](\tau, \bar{t}, \bar{x}), \\
\delta & =\delta_{s}\left[z^{(m)}, u_{s}^{(m+1)}\right](t, x), & \bar{\delta} & =\delta_{s}\left[z^{(m)}, u_{s}^{(m+1)}\right](\bar{t}, \bar{x}),
\end{aligned}
$$

and

$$
Q(\theta, \tau)=\theta \bar{P}(\tau)+(1-\theta) P(\tau), \quad W^{(m)}(\tau)=W_{s}^{(m)}\left[u_{s}^{(m+1)}\right](\tau, t, x) .
$$


It follows from (21) and (22) that

$$
\begin{aligned}
\Delta^{(m)}(t, \bar{t}, x, \bar{x})= & F_{s}\left[z^{(m)}, u_{s}^{(m+1)}\right](\bar{t}, \bar{x})-F_{s}\left[z^{(m)}, u_{s}^{(m+1)}\right](t, x) \\
& -u_{0, s}^{(m+1)}(t, x)(\bar{t}-t)-G_{s}^{(m)}\left[u^{(m+1)}\right](t, x) \circ(\bar{x}-x) .
\end{aligned}
$$

To simplify the formulation of properties of the function $\Delta^{(m)}$ we define

$$
\begin{aligned}
A^{(m)}(t, \bar{t}, x, \bar{x})= & \varphi_{s}(\bar{\delta}, \bar{g}(\bar{\delta}))-\varphi_{s}(\delta, g(\delta)) \\
& -\partial_{t} \varphi_{s}(\delta, g(\delta))(\bar{\delta}-\delta)-\partial_{x} \varphi_{s}(\delta, g(\delta)) \circ[\bar{g}(\bar{\delta})-g(\delta)], \\
B^{(m)}(t, \bar{t}, x, \bar{x})= & \partial_{t} \varphi_{s}(\delta, g(\delta))(\bar{\delta}-\delta) \\
& +\partial_{x} \varphi_{s}(\delta, g(\delta)) \circ[\bar{g}(\bar{\delta})-g(\delta)-\bar{x}+x], \\
C^{(m)}(\tau, t, \bar{t}, x, \bar{x})= & \int_{\tau}^{t}\left[\partial_{q} f_{s}(\bar{P}(\xi))-\partial_{q} f_{s}(P(\xi))\right] d \xi+\int_{t}^{\bar{t}} \partial_{q} f_{s}(\bar{P}(\xi)) d \xi, \\
\Lambda^{(m)}(t, \bar{t}, x, \bar{x})= & \left(\int_{\bar{\delta}}^{\delta}+\int_{t}^{\bar{t}}\right)\left[f_{s}(\bar{P}(\tau))-u_{s}^{(m+1)}(\tau, \bar{g}(\tau)) \circ \partial_{q} f_{s}(\bar{P}(\tau))\right] d \tau .
\end{aligned}
$$

It follows from (32) that

$$
\begin{aligned}
& \Delta^{(m)}(t, \bar{t}, x, \bar{x})=\varphi_{s}(\bar{\delta}, \bar{g}(\delta))-\varphi_{s}(\delta, g(\delta))+\int_{\delta}^{t}\left[f_{s}(\bar{P}(\tau))-f_{s}(P(\tau))\right] d \tau \\
& \quad+\int_{\delta}^{t}\left[-u_{s}^{(m+1)}(\tau, \bar{g}(\tau)) \circ \partial_{q} f_{s}(\bar{P}(\tau))+u_{s}^{(m+1)}(\tau, g(\tau)) \circ \partial_{q} f_{s}(P(\tau))\right] d \tau \\
& \quad+\Lambda^{(m)}(t, \bar{t}, x, \bar{x})-u_{0, s}^{(m+1)}(t, x)(\bar{t}-t)-G_{s}^{(m)}\left[u^{(m+1)}\right](t, x) \circ(\bar{x}-x) .
\end{aligned}
$$

Having disposed of this preliminary step, we apply the Hadamard mean value theorem to the difference

$$
f_{s}(P(\tau))-f_{s}(\bar{P}(\tau)) .
$$

We thus get

$$
\Delta^{(m)}(t, \bar{t}, x, \bar{x})=\bar{\Delta}^{(m)}(t, \bar{t}, x, \bar{x})+\widetilde{\Delta}^{(m)}(t, \bar{t}, x, \bar{x})
$$

where

$$
\begin{aligned}
\bar{\Delta}^{(m)}(t, \bar{t}, x, \bar{x}) & =A^{(m)}(t, \bar{t}, x, \bar{x}) \\
& +\int_{\delta}^{t} \int_{0}^{1}\left[\partial_{x} f_{s}(Q(\theta, \tau))-\partial_{x} f_{s}(P(\tau))\right] d \theta \circ[\bar{g}(\tau)-g(\tau)] d \tau \\
& +\int_{\delta}^{t} \int_{0}^{1}\left[\partial_{w} f_{s}(Q(\theta, \tau))-\partial_{w} f_{s}(P(\tau))\right] d \theta\left[z_{\alpha(\tau, \bar{g}(\tau))}^{(m)}-z_{\alpha(\tau, g(\tau))}^{(m)}\right] d \tau
\end{aligned}
$$




$$
\begin{aligned}
& +\int_{\delta}^{t}\left[u_{s}^{(m+1)}(\tau, \bar{g}(\tau))-u_{s}^{(m+1)}(\tau, g(\tau))\right] \\
& \circ \int_{0}^{1}\left[\partial_{q} f_{s}(Q(\theta, \tau))-\partial_{q} f_{s}(\bar{P}(\tau))\right] d \theta d \tau \\
& +\int_{\delta}^{t} \partial_{w} f_{s}(P(\tau))\left[z_{\alpha(\tau, \bar{g}(\tau))}^{(m)}-z_{\alpha(\tau, g(\tau))}^{(m)}-W^{(m)}(\tau) \circ(\bar{g}(\tau)-g(\tau))\right] d \tau
\end{aligned}
$$

and

$$
\begin{aligned}
& \widetilde{\Delta}^{(m)}(t, \bar{t}, x, \bar{x})=B^{(m)}(t, \bar{t}, x, \bar{x}) \\
& +\int_{\delta}^{t}\left[\partial_{x} f_{s}(P(\tau))+\partial_{w} f_{s}(P(\tau)) W^{(m)}(\tau)\right] \circ[\bar{g}(\tau)-g(\tau)-\bar{x}+x] d \tau \\
& \quad-\int_{\delta}^{t} u_{s}^{(m+1)}(\tau, g(\tau)) \circ\left[\partial_{q} f_{s}(\bar{P}(\tau))-\partial_{q} f_{s}(P(\tau))\right] d \tau \\
& +\Lambda^{(m)}(t, \bar{t}, x, \bar{x})-u_{0, s}^{(m+1)}(t, x)(\bar{t}-t) .
\end{aligned}
$$

Let us estimate $\left|\widetilde{\Delta}^{(m)}(t, \bar{t}, x, \bar{x})\right|$. It follows from (11) that

$$
\bar{g}(\tau)-g(\tau)-\bar{x}+x=C^{(m)}(\tau, t, \bar{t}, x, \bar{x})
$$

and

$$
\bar{g}(\bar{\delta})-g(\delta)-\bar{x}+x=\left(\int_{\bar{\delta}}^{\delta}+\int_{t}^{\bar{t}}\right) \partial_{q} f_{s}(\bar{P}(\tau)) d \tau+\int_{\delta}^{t}\left[\partial_{q} f_{s}(\bar{P}(\tau))-\partial_{q} f_{s}(P(\tau))\right] d \tau .
$$

Substituting the above relations into $\widetilde{\Delta}^{(m)}(t, \bar{t}, x, \bar{x})$ and changing the order of integrals where necessary we obtain

$$
\begin{aligned}
\widetilde{\Delta}^{(m)}(t, \bar{t}, x, \bar{x})= & D^{(m)}(t, \bar{t}, x, \bar{x}) \\
& +\int_{\delta}^{t} E^{(m)}(\tau, t, x) \circ\left[\partial_{q} f_{s}(\bar{P}(\tau))-\partial_{q} f_{s}(P(\tau))\right] d \tau,
\end{aligned}
$$

where

$$
\begin{aligned}
D^{(m)}(t, \bar{t}, x, \bar{x})= & \int_{t}^{\bar{t}}\left[\left(u_{s}^{(m+1)}(t, x)-u_{s}^{(m+1)}(\tau, \bar{g}(\tau))\right) \circ \partial_{q} f_{s}(\bar{P}(\tau))\right. \\
& \left.+f_{s}(\bar{P}(\tau))-u_{0, s}^{(m+1)}(t, x)\right] d \tau \\
& +\int_{\bar{\delta}}^{\delta}\left[\left(\partial_{x} \varphi_{s}(\delta, g(\delta))-u_{s}^{(m+1)}(\tau, \bar{g}(\tau))\right) \circ \partial_{q} f_{s}(\bar{P}(\tau))\right. \\
& \left.+f_{s}(\bar{P}(\tau))-\partial_{t} \varphi_{s}(\delta, g(\delta))\right] d \tau
\end{aligned}
$$


and

$$
\begin{aligned}
E^{(m)}(\tau, t, x)= & -u_{s}^{(m+1)}(\tau, g(\tau))+\partial_{x} \varphi_{s}(\delta, g(\delta)) \\
& +\int_{\delta}^{\tau}\left[\partial_{x} f_{s}(P(\xi))+\partial_{w} f_{s}(P(\xi)) W^{(m)}(\xi)\right] d \xi
\end{aligned}
$$

The next claim is that $E(\tau, t, x)=0$ for $(t, x),(\bar{t}, \bar{x}) \in E_{c}$ and $\tau \in[0, c]$. To see this, write $(21)$ at the point $(\tau, g(\tau))$ and note that

$$
g_{s}\left[z^{(m)}, u_{s}^{(m+1)}\right](\xi, \tau, g(\tau))=g_{s}\left[z^{(m)}, u_{s}^{(m+1)}\right](\xi, t, x)=g(\xi) .
$$

Thus $\widetilde{\Delta}^{(m)}(t, \bar{t}, x, \bar{x})=D^{(m)}(t, \bar{t}, x, \bar{x})$. We conclude from (22) and Lemma 2.1 that there is $C_{1} \in \mathbb{R}_{+}$such that

$$
\left|\widetilde{\Delta}^{(m)}(t, \bar{t}, x, \bar{x})\right| \leq C_{1}[|\bar{t}-t|+\|\bar{x}-x\|]^{2} .
$$

We will now estimate $\bar{\Delta}^{(m)}(t, \bar{t}, x, \bar{x})$. It follows from Assumption $\mathrm{H}[f]$ that

$$
\left\|\partial_{x} f_{s}(Q(s, \tau))-\partial_{x} f_{s}(P(\tau))\right\| \leq L \bar{d}\|\bar{g}(\tau)-g(\tau)\|
$$

and similar estimates hold for $\partial_{w} f_{s}, \partial_{q} f_{s}$. An easy computation shows that

$$
\begin{aligned}
& \left|A^{(m)}(t, \bar{t}, x, \bar{x})\right| \leq s_{2}(|\bar{\delta}-\delta|+\|\bar{g}(\bar{\delta})-g(\delta)\|)^{2}, \\
& \left\|z_{\alpha(\tau, \bar{g}(\tau))}^{(m)}-z_{\alpha(\tau, g(\tau))}^{(m)}\right\|_{D} \leq d r_{0}\|\bar{g}(\tau)-g(\tau)\|, \\
& \left\|u_{s}^{(m+1)}(\tau, \bar{g}(\tau))-u_{s}^{(m+1)}(\tau, g(\tau))\right\| \leq p_{1}\|\bar{g}(\tau)-g(\tau)\| .
\end{aligned}
$$

Since $\partial z^{(m)}=\left(u_{0}^{(m)}, u^{(m)}\right)$ on $E_{c}^{*}$, we have

$$
\left\|z_{\alpha(\tau, \bar{g}(\tau))}^{(m)}-z_{\alpha(\tau, g(\tau))}^{(m)}-W^{(m)}(\tau) \circ[\bar{g}(\tau)-g(\tau)]\right\|_{D} \leq n \bar{\omega}\|\bar{g}(\tau)-g(\tau)\|^{2} .
$$

The above estimates and the definition of $\bar{\Delta}^{(m)}(t, \bar{t}, x, \bar{x})$ yield

$$
\bar{\Delta}^{(m)}(t, \bar{t}, x, \bar{x}) \leq s_{2}(|\bar{\delta}-\delta|+\|\bar{g}(\bar{\delta})-g(\delta)\|)^{2}+A^{*} \int_{\delta}^{t}\|\bar{g}(\tau)-g(\tau)\|^{2} d \tau,
$$

where $A^{*}=B n \bar{\omega}+L \bar{d}^{2}$. It follows from Lemma 2.1 that there is $C_{2} \in \mathbb{R}_{+}$ such that

$$
\bar{\Delta}^{(m)}(t, \bar{t}, x, \bar{x}) \leq C_{2}[|\bar{t}-t|+\|\bar{x}-x\|]^{2} .
$$

Adding inequalities (33) and (35), we get (31), and from arbitrariness of $(t, x),(\bar{t}, \bar{x}) \in E_{c}$, and $s \in \mathcal{S}$,

$$
\partial z^{(m+1)}=\left(u_{0}^{(m+1)}, u^{(m+1)}\right) \quad \text { on } E_{c} .
$$

This completes the proof of $(29)$.

We now prove that $z^{(m+1)} \in C_{\varphi . c}^{L}[d]$. It is clear that $z^{(m+1)}$ is continuous on $E_{c}$ and $z^{(m+1)}=\varphi$ on $E_{0} \cup \partial_{0} E$. Moreover, it follows from Assumption $\mathrm{H}[c, d, p]$ and $(29)$ that $\left\|z^{(m+1)}(t, x)-z^{(m+1)}(\bar{t}, \bar{x})\right\| \leq d[|\bar{t}-t|+\|\bar{x}-x\|]$. 
It remains to prove $u_{0}^{(m+1)} \in C_{\varphi . c}^{L}[p ; 1]$. Clearly, $u_{0}^{(m+1)}$ is continuous on $E_{c}$ and $u_{0}^{(m+1)}=\partial_{t} \varphi$ on $E_{0} \cup \partial_{0} E$. Furthermore, Assumption $\mathrm{H}[c, d, p]$ implies $\left\|u_{0}^{(m+1)}(t, x)\right\| \leq p_{0}$ and $\left\|u_{0}^{(m+1)}(t, x)-u_{0}^{(m+1)}(\bar{t}, \bar{x})\right\| \leq p_{1}[|\bar{t}-t|+$ $\|\bar{x}-x\|]$.

Now we prove that the sequence $\left\{\left(z, u_{0}, u\right)^{(m)}\right\}$ is uniformly convergent on $E_{c}$.

Lemma 3.6. If Assumptions $\mathrm{H}[\alpha], \mathrm{H}[f]$ and $\mathrm{H}[c]$ are satisfied, then the sequence $\left\{\left(z, u_{0}, u\right)^{(m)}\right\}$ is uniformly convergent on $E_{c}$.

Proof. For $t \in[0, c]$ and $m \geq 1$, we set

$$
Z^{(m)}(t)=\left\|z^{(m)}-z^{(m-1)}\right\|_{t}, \quad U^{(m)}(t)=\left\|u^{(m)}-u^{(m-1)}\right\|_{(t)} .
$$

It follows from Lemma 2.1 that

$$
\begin{aligned}
&\left\|g\left[z^{(m)}, u^{(m+1)}\right](\tau, t, x)-g\left[z^{(m-1)}, u^{(m)}\right](\tau, t, x)\right\| \\
& \leq \bar{C}\left|\int_{t}^{\tau}\left[Z^{(m)}(\xi)+U^{(m+1)}(\xi)\right] d \xi\right|
\end{aligned}
$$

and

$$
\begin{aligned}
\left|\delta_{s}\left[z^{(m)}, u_{s}^{(m+1)}\right](t, x)-\delta_{s}\left[z^{(m-1)}, u_{s}^{(m)}\right](t, x)\right| & \\
& \leq \widehat{C} \int_{0}^{t}\left[Z^{(m)}(\tau)+U^{(m+1)}(\tau)\right] d \tau, \quad s \in \mathcal{S} .
\end{aligned}
$$

Hence we obtain the integral inequality

$$
\begin{aligned}
U^{(m+1)}(t) \leq & \widetilde{A} \int_{0}^{t} U^{(m+1)}(\tau) d \tau \\
& +\int_{0}^{t}\left[\widetilde{B} Z^{(m-1)}(\tau)+\widetilde{A} Z^{(m)}(\tau)+\widetilde{B} U^{(m)}(\tau)\right] d \tau
\end{aligned}
$$

for some constants $\widetilde{A}, \widetilde{B} \in \mathbb{R}_{+}$, independent of $m$. The above estimate and the Gronwall inequality yield

$$
U^{(m+1)}(t) \leq e^{\widetilde{A} c} \int_{0}^{t}\left[\widetilde{B} Z^{(m-1)}(\tau)+\widetilde{A} Z^{(m)}(\tau)+\widetilde{B} U^{(m)}(\tau)\right] d \tau, \quad m \geq 1 .
$$

An easy computation shows that there is a constant $\bar{B} \in \mathbb{R}_{+}$independent of $m$, such that

$$
Z^{(m+1)}(t) \leq \bar{B}_{0}^{t}\left[Z^{(m)}(\tau)+U^{(m+1)}(\tau)\right] d \tau
$$


The above two inequalities yield

$$
\begin{aligned}
Z^{(m+1)}(t)+U^{(m+1)}(t) \leq & D_{1} \int_{0}^{t}\left[Z^{(m)}(\tau)+U^{(m)}(\tau)\right] d \tau \\
& +D_{2} \int_{0}^{t} Z^{(m-1)}(\tau) d \tau
\end{aligned}
$$

for all $m \geq 1$ and some $D_{1}, D_{2} \in \mathbb{R}_{+}$. We define the norm in the space $C([0, c], \mathbb{R})$ as follows:

$$
\|\zeta\|_{\lambda}=\max \left\{|\zeta(t)| e^{-\lambda t}: t \in[0, c]\right\},
$$

where $\lambda>D_{1}+D_{2}$. According to (36), we have

$$
\begin{aligned}
Z^{(m+1)}(t) & +U^{(m+1)}(t) \\
& \leq D_{1} \int_{0}^{t}\left[Z^{(m)}(\tau)+U^{(m)}(\tau)\right] d \tau+D_{2} \int_{0}^{t} Z^{(m-1)}(\tau) d \tau \\
& =D_{1} \int_{0}^{t}\left[Z^{(m)}(\tau)+U^{(m)}(\tau)\right] e^{-\lambda \tau} e^{\lambda \tau} d \tau+D_{2} \int_{0}^{t} Z^{(m-1)}(\tau) e^{-\lambda \tau} e^{\lambda \tau} d \tau \\
& \leq D_{1}\left\|Z^{(m)}+U^{(m)}\right\|_{\lambda} \int_{0}^{t} e^{\lambda \tau} d \tau+D_{2}\left\|Z^{(m-1)}\right\|_{\lambda} \int_{0}^{t} e^{\lambda \tau} d \tau \\
& =\frac{D_{1}}{\lambda}\left\|Z^{(m)}+U^{(m)}\right\|_{\lambda}\left(e^{\lambda t}-1\right)+\frac{D_{2}}{\lambda}\left\|Z^{(m-1)}\right\|_{\lambda}\left(e^{\lambda t}-1\right) \\
& \leq\left(\frac{D_{1}}{\lambda}\left\|Z^{(m)}+U^{(m)}\right\|_{\lambda}+\frac{D_{2}}{\lambda}\left\|Z^{(m-1)}\right\|_{\lambda}\right) e^{\lambda t}
\end{aligned}
$$

for $t \in[0, c]$. It follows that

$$
\left|Z^{(m+1)}(t)+U^{(m+1)}(t)\right| e^{-\lambda t} \leq \frac{D_{1}}{\lambda}\left\|Z^{(m)}+U^{(m)}\right\|_{\lambda}+\frac{D_{2}}{\lambda}\left\|Z^{(m-1)}\right\|_{\lambda}
$$

for $t \in[0, c]$. Writing $y^{(m)}=\left\|Z^{(m)}+U^{(m)}\right\|_{\lambda}$ yields

$$
0 \leq y^{(m+1)} \leq \gamma_{1} y^{(m)}+\gamma_{2} y^{(m-1)} .
$$

Moreover, from Lemmata 3.3 and 3.5 we get $y^{(1)}, y^{(2)} \leq 2(d \bar{b}+\widetilde{p})$, where $\widetilde{p}=\min \left\{p_{0}, p_{1} \bar{b}\right\}$ and $\bar{b}=\min \{b, c\}$. From the above and from the stability theory for difference equations (using the fact that $\gamma_{1}+\gamma_{2}<1$ ), there exist $C \in \mathbb{R}_{+}$and $q \in(0,1)$ such that

$$
y^{(m)} \leq C q^{m} \quad \text { for } m \in \mathbb{N} .
$$


By the above inequality, $\left\{z^{(m)}\right\},\left\{u^{(m)}\right\}$ are Cauchy sequences in $C_{\varphi . c}^{L}[d]$, $C_{\varphi . c}^{L}[p ; n]$, hence are uniformly convergent. Now, define

$$
U_{0}^{(m)}(t)=\left\|u_{0}^{(m)}-u_{0}^{(m-1)}\right\|_{t} .
$$

Note that from (22) we get

$$
U_{0}^{(m+1)}(t) \leq B\left[Z^{(m)}(t)+U^{(m+1)}(t)\right] \leq B C(1+q) q^{m} .
$$

Since Lemma 3.3 implies $U_{0}^{(1)}(t) \leq 2 \widetilde{p},\left\{u_{0}^{(m)}\right\}$ is a Cauchy sequence (in $\left.C_{\varphi . c}^{L}[p ; 1]\right)$ as well, and hence is uniformly convergent.

4. Existence of solutions of nonlinear mixed problems. We can now state the main result on the existence of classical solutions to problem (1), (2). For a function $\varphi \in C^{1 . L}[s]$ and $\tau \in[0, a]$ we write

$$
\begin{aligned}
\|\varphi\|_{\tau} & =\max \left\{\|\varphi(t, x)\|:(t, x) \in E_{0} \cup \partial_{0} E_{\tau}\right\}, \\
\left\|\partial_{t} \varphi\right\|_{\tau} & =\max \left\{\left\|\partial_{t} \varphi(t, x)\right\|:(t, x) \in E_{0} \cup \partial_{0} E_{\tau}\right\}, \\
\left\|\partial_{x} \varphi\right\|_{\tau} & =\max \left\{\left\|\partial_{x} \varphi(t, x)\right\|:(t, x) \in E_{0} \cup \partial_{0} E_{\tau}\right\} .
\end{aligned}
$$

TheOREM 4.1. If Assumptions $\mathrm{H}[\alpha], \mathrm{H}[f]$ and $\mathrm{H}[c]$ are satisfied, then, for every $\varphi \in C^{1 . L}[s]$, there exists a solution $v: E_{c}^{*} \rightarrow X$ of problem (1), (2). Moreover, $v \in C_{\varphi . c}^{L}[d]$ and $\partial v \in C_{\varphi . c}^{L}[p ; 1+n]$.

If $\bar{\varphi} \in C^{1 . L}[s]$ and $\bar{v} \in C_{\bar{\varphi} . c}^{L}[d]$ is a solution of system (1) with initial boundary condition $z=\bar{\varphi}$ on $E_{0} \cup \partial_{0} E$, then there is $\Lambda_{c} \in \mathbb{R}_{+}$such that for $0 \leq t \leq c$,

$$
\begin{aligned}
\|\bar{v}-v\|_{t}+\left\|\partial_{t} v-\partial_{t} \bar{v}\right\|_{(t)}+\left\|\partial_{x} v-\partial_{x} \bar{v}\right\|_{(t)} \\
\quad \leq \Lambda_{c}\left[\|\bar{\varphi}-\varphi\|_{t}+\left\|\partial_{t} \varphi-\partial_{t} \bar{\varphi}\right\|_{t}+\left\|\partial_{x} \varphi-\partial_{x} \bar{\varphi}\right\|_{t}\right] .
\end{aligned}
$$

Proof. It follows from Lemmata 3.5 and 3.6 that there is $v \in C_{\varphi . c}^{L}[d]$ such that

$$
v(t, x)=\lim _{m \rightarrow \infty} z^{(m)}(t, x), \quad \partial z(t, x)=\lim _{m \rightarrow \infty} u^{(m)}(t, x)
$$

uniformly on $E_{c}$. Thus, we get

$$
v(t, x)=F\left[v, \partial_{x} v\right](t, x), \quad \partial_{x} v(t, x)=G\left[v, \partial_{t} v, \partial_{x} v\right](t, x),
$$

and

$$
g_{s}\left[v, \partial_{x} v_{s}\right](\tau, t, x)=x+\int_{\tau}^{t} \partial_{q} f_{s}\left(P_{s}\left[v, \partial_{x} v_{s}\right](\xi, t, x)\right) d \xi .
$$

Moreover, the initial boundary conditions

$$
v=\varphi, \quad \partial v=\partial \varphi \quad \text { on } E_{0} \cup \partial_{0} E_{c}
$$

are satisfied. It follows that $v$ is a classical solution of problem (1), (2) on $E_{c}^{*}$. The proof is similar to the proof of the corresponding properties 
for initial-value or initial boundary value problems considered in [3] and [8] (Chapter 4). The details are omitted.

We now prove (37). To this end, we first prove

$$
\|\bar{v}-v\|_{t}+\left\|\partial_{x} v-\partial_{x} \bar{v}\right\|_{(t)} \leq \bar{\Lambda}_{c}\left[\|\bar{\varphi}-\varphi\|_{t}+\left\|\partial_{t} \varphi-\partial_{t} \bar{\varphi}\right\|_{t}+\left\|\partial_{x} \varphi-\partial_{x} \bar{\varphi}\right\|_{t}\right]
$$

for $0 \leq t \leq c$. The functions $\left(\bar{v}, \partial_{x} \bar{v}\right)$ satisfy the integral functional system (16), (17) and the initial boundary condition (18) with $\bar{\varphi}$ instead of $\varphi$. It easily follows that there are $\Lambda_{0}, \Lambda_{1} \in \mathbb{R}_{+}$such that

$$
\begin{aligned}
\|\bar{v}-v\|_{t}+\left\|\partial_{x} v-\partial_{x} \bar{v}\right\|_{(t)} & \leq \Lambda_{0}\left[\|\bar{\varphi}-\varphi\|_{t}+\left\|\partial_{t} \varphi-\partial_{t} \bar{\varphi}\right\|_{t}+\left\|\partial_{x} \varphi-\partial_{x} \bar{\varphi}\right\|_{t}\right] \\
& +\Lambda_{1} \int_{0}^{t}\left[\|\bar{v}-v\|_{\tau}+\left\|\partial_{x} v-\partial_{x} \bar{v}\right\|_{(\tau)}\right] d \tau, \quad 0 \leq t \leq c .
\end{aligned}
$$

Using the Gronwall inequality, we get (38) with $\bar{\Lambda}_{c}=\Lambda_{0} \exp \left(\Lambda_{1} c\right)$. Moreover, from (17) we have

$$
\left\|\partial_{t} v-\partial_{t} \bar{v}\right\|_{(t)} \leq\left\|\partial_{t} \varphi-\partial_{t} \bar{\varphi}\right\|_{t}+B\left[\|\bar{v}-v\|_{t}+\left\|\partial_{x} v-\partial_{x} \bar{v}\right\|_{(t)}\right], \quad 0 \leq t \leq c,
$$

which, together with (38), yields (37) with $\Lambda_{c}=1+(1+B) \bar{\Lambda}_{c}$.

REMARK 4.2. Note that a corresponding existence result for systems of equations with deviated argument, or integral-differential equations, of the form given in Examples 1.1, 1.2, may be easily obtained from the preceding theorem.

REMARK 4.3. The theory presented in this paper may be easily extended to systems of equations with several function variables:

$$
\partial_{t} z_{s}(t, x)=f_{s}\left(t, x, z_{\alpha_{1}(t, x)}, z_{\alpha_{2}(t, x)}, \ldots, z_{\alpha_{n}(t, x)}, \partial_{x} z_{s}(t, x)\right), \quad s \in \mathcal{S},
$$

with the initial-boundary condition (2). Such a model would allow one to consider mixed problems with both deviated argument and a classical argument:

$$
\partial_{t} z_{s}(t, x)=f_{s}\left(t, x, z_{\alpha(t, x)}, z(t, x), \partial_{x} z_{s}(t, x)\right), \quad s \in \mathcal{S},
$$

and to obtain existence results for them.

\section{References}

[1] P. Brandi and R. Ceppitelli, On the existence of the solution of a nonlinear functional partial differential equations of the first order, Atti Sem. Mat. Fis. Univ. Modena 29 (1980), 166-186.

[2] - - - Existence, uniqueness and continuous dependence for a hereditary nonlinear functional partial differential equation of the first order, Ann. Polon. Math. 47 (1986), 121-136.

[3] W. Czernous, Generalized solutions of mixed problems for first order partial functional differential equations, Ukrainian Math. J. 58 (2006), 904-936. 
[4] T. Człapiński, On the existence of generalized solutions of nonlinear first order partial differential-functional equations in two independent variables, Czechoslovak Math. J. 41 (116) (1991), 490-506.

[5] —, Generalized solutions to boundary value problems for quasilinear hyperbolic systems of partial differential functional equations, Ann. Polon. Math. 57 (1992), 177191.

[6] M. I. Imanaliev and Yu. A. Ved', A first-order partial differential equation with an integral coefficient, Differentsial'nye Uravneniya 25 (1989), 465-477, 549 (in Russian); English transl.: Differential Equations 25 (1989), 325-335.

[7] Z. Kamont, Initial value problems for hyperbolic differential-functional systems, Boll. Un. Mat. Ital. B (7) 8 (1994), 965-984.

[8] - Hyperbolic Functional Differential Inequalities and Applications, Math. Appl. 486, Kluwer, Dordrecht, 1999.

[9] —, Infinite systems of hyperbolic functional differential inequalities, Nonlinear Anal. 51 (2002), 1429-1445.

[10] —, Infinite systems of hyperbolic functional differential equations, Ukrainian Math. J. 55 (2003), 2006-2030.

[11] H. Leszczyński, On the existence and uniqueness of weak solutions of the Cauchy problem for weakly coupled systems of nonlinear partial differential-functional equations of the first order, Boll. Un. Mat. Ital. B (7) 7 (1993), 323-354.

[12] A. D. Myshkis and A. S. Šlopak, A mixed problem for systems of differentialfunctional equations with partial derivatives and with operators of Volterra type, Mat. Sb. 41 (1957), 239-256 (in Russian).

[13] A. Salvadori, The Cauchy problem for a hereditary hyperbolic structure. Existence, uniqueness and continuous dependence, Atti Sem. Mat. Fis. Univ. Modena 32 (1983), 329-356.

[14] J. Szarski, Cauchy problem for an infinite system of differential-functional equations with first order partial derivatives, Comment. Math. Special Issue 1 (1978), 293-300.

[15] —, Infinite systems of first-order partial-differential functional inequalities, in: General Inequalities, 2 (Oberwolfach, 1978), Birkhäuser, Basel, 1980, 121-126.

[16] J. Turo, Mixed problems for quasilinear hyperbolic systems, in: Proceedings of the Second World Congress of Nonlinear Analysts, Part 4 (Athens, 1996), Nonlinear Anal. 30 (1997), 2329-2340.

[17] T. Ważewski, Sur le problème de Cauchy relatif à un système d'équations aux dérivées partielles, Ann. Soc. Polon. Math. 15 (1936), 101-127.

[18] J. Wu, Theory and Applications of Partial Functional Differential Equations, Springer, New York, 1996.

Institute of Mathematics

University of Gdańsk

Wita Stwosza 57

80-952 Gdańsk, Poland

E-mail: Wojciech.Czernous@math.univ.gda.pl

Received 24.10.2007

and in final form 23.6.2008 